\title{
Genome editing in clinical genetics: points to consider-a statement of the American College of Medical Genetics and Genomics
}

\author{
ACMG Board of Directors ${ }^{1}$
}

Disclaimer: These recommendations are designed primarily as an educational resource for medical geneticists and other health-care providers, to help them provide quality medical genetic services. Adherence to these recommendations does not necessarily assure a successful medical outcome. These recommendations should not be considered inclusive of all proper procedures and tests or exclusive of other procedures and tests that are reasonably directed to obtaining the same results. In determining the propriety of any specific procedure or test, the geneticist should apply his or her own professional judgment to the specific clinical circumstances presented by the individual patient or specimen. It may be prudent, however, to document in the patient's record the rationale for any significant deviation from these recommendations.

Genet Med advance online publication 26 January 2017

Key Words: biotechnology; CRISPR/Cas9; gene editing; genetic engineering; gene drives
Medical geneticists provide diagnosis, counseling, management, and treatment for individuals and families affected by genetic disorders. These disorders are due to genetic variations that may range from gain or loss of entire chromosomes to alterations involving only a single DNA base pair. Management options have typically included anticipatory guidance, surveillance for complications, surgery, dietary management, medications, and, in some recent instances, gene replacement therapy. Progress in these areas has brought comfort, hope, and relief to many patients and families who live with genetic conditions, some of which have devastating effects on health and well-being.

Because the underlying causes of these conditions may be changes in the structure of a gene or a region of the genome, the question has been raised as to whether it is possible to alter the genetic code in an affected individual to alleviate the pathology. In principle, this could be done in somatic cells to restore function at the tissue level, or it could be done in the embryo, both to treat that individual and to remove the variant from the germline of that individual. Until recently, this kind of approach was technologically out of reach, but with the advent of genome editing approaches, especially CRISPR/Cas9, it is becoming increasingly feasible. CRISPR/Cas9 is an RNA-guided nuclease system of bacterial origin that can be engineered to target a specific sequence in the genome where the Cas9 protein causes a precise double-strand break. Subsequent DNA repair by the cellular machinery results in either imprecise repair by the nonhomologous end-joining or precise repair by template-driven homology-directed repair. Genome editing is an area of very rapid technological change, so what is not possible today could well become a reality in the very near future. As a consequence, although the American College of Medical Genetics and Genomics (ACMG) is focused on current clinical practice, the ACMG Board of Directors feels compelled to issue these points to consider regarding the potential clinical application of genome editing.

\section{Points to consider}

1. ACMG applauds the research applications of genome editing technologies, which are proving to be of great value in developing disease models and studying disease mechanisms. However, the current limitations in these technologies-such as off-target effects-must be overcome prior to any clinical application.

2. Application of genome editing technologies to alter pathogenic variants in somatic cells offers promise in the treatment of individuals with disorders due to single-gene variants that primarily affect specific tissues, such as liver or blood cells. As with any new clinical intervention, clinical application of genome editing technology will require stringent medical and genetic review. Among the concerns that must be addressed are the needs to ensure that:

a. The underlying pathogenic variant has been corrected to a form that will not be pathogenic.

${ }^{1}$ American College of Medical Genetics and Genomics, Bethesda, Maryland, USA. Correspondence: Michael S. Watson (mwatson@acmg.net)

The Board of Directors of the American College of Medical Genetics and Genomics approved this statement on 18 October 2016. 
b. No other genetic variations have been introduced in the process of editing the pathogenic variant.

c. The cells that have been edited have not acquired other genetic variants as part of the process of treatment, for example, during cell culture.

d. The cells treated do not have epigenetic marks that will result in abnormal function if transplanted back into an individual.

3. Application of genome editing at the level of the embryo raises many technical and ethical concerns, including:

a. The risk of off-target effects of genome editing may have unpredictable consequences to the embryo and, because the germ line is involved, to future generations as well. Any potential adverse effects could have far-reaching consequences that could take years or even decades to recognize.

b. The consequences of editing a pathogenic variant may have unknown epigenetic effects that may alter normal patterns of gene expression in some tissues.

c. The decision as to which specific genetic variants should be subject to genome editing needs further discussion at a societal level. Some variants that are associated with highly penetrant disorders with major adverse effects on health and quality of life might seem like compelling candidates for therapeutic editing. It is inevitable, however, that consideration will also be given to editing variants associated with phenotypes that are not fully penetrant and for which effects on quality of life are less clear. Ultimately, one can foresee efforts to edit variants that are associated with nondisease traits or contribute to multifactorial disorders in unpredictable ways. Such issues are not typically of concern in the management of children or adults with genetic conditions, but will become critical if gene editing in the embryo is contemplated.

In light of these potentially serious and far-reaching concerns, the ACMG Board of Directors believes that genome editing in the human embryo is premature and should be subject to vigorous ethical debate and further refinement of technological issues.

\section{CONCLUSION}

Genome editing offers great promise for the future treatment of individuals and families with genetic disorders, but also raises major technological and ethical issues that must be resolved before clinical application. The potential for rapid advance of this approach, and the pressure to apply it clinically, should not be underestimated. The ACMG Board of Directors strongly encourages broad public debate regarding the clinical application of genomic editing and will appoint an ad hoc committee to recommend specific areas where it can contribute to this debate.

\section{DISCLOSURE}

The authors declare no conflict of interest. 